\title{
BPH: Risikopatienten mit Alphablockern und 5-ARI behandeln
}

- Die benigne Prostatahyperplasie (BPH) ist eine progressive Erkrankung. Innerhalb von vier Jahren verschlechtern sich bei $31 \%$ der Patienten die Symptome von leicht bis moderat zu schwer (IPS-Score $\geq 19$ ). Um Langzeitfolgen wie einen akuten Harnverhalt (AUR) und eine Operation zu vermeiden, müssen frühzeitig Risikofaktoren identifiziert werden. Laut Prof. Michael Marberger, Wien, zählen dazu Alter, reduzierte Harndurchflussrate $(\mathrm{Qmax})$, Prostatavolumen $>30$ $\mathrm{cm}^{3}$ und PSA-Wert $>1,6 \mathrm{ng} / \mathrm{ml}$. Bei der Diagnostik könne der PSA-Wert gemäß der USamerikanischen, kanadischen und europäischen Leitlinien als Surrogatparameter für die Prostatagröße verwendet werden, so Marberger.

Mit einer Alphablocker-Monotherapie können zwar rasch die Symptome gelindert werden, das AUR-Risiko besteht aber nach wie vor und ist umso höher, je später ein 5-Alpha-Reduktase-Hemmer (5-ARI) verschrieben wird [Naslund et al. Curr Med Res
Opin 2009; 25: 2663-9]. Deshalb sollten gerade Risiko-Patienten frühzeitig mit einer Kombination aus Alphablocker und 5-ARI behandelt werden, empfahl Prof. Mark Emberton, London. Die Fixkombination aus Dutasterid und Tamsulosin (Duodart ${ }^{\oplus}$ ) ist dabei die einzige Option mit Evidenz 1b, die einen Langzeitvorteil für Patienten mit Progressionsrisiko bietet. Mit der Kombination lässt sich die Zeit bis zu einem BPH-Progress und einem AUR oder einer mit BPH assoziierten Operation signifikant länger herauszögern und die Symptome bessern sich stärker als mit einer Dutasterid- oder Tamsulosin-Monotherapie.

Die häufigsten Nebenwirkungen der Kombinationstherapie sind sexueller Art (u.a. erektile Dysfunktion, Libidostörungen). Die schwersten sexuellen Nebenwirkungen unter 5-ARI treten im ersten Jahr auf und werden dann seltener. Prof. Gerald Andriole, St. Louis/ USA, spekulierte, dass ein „Nocebo"-Effekt für diese Häufung verantwortlich sein könnte.
So berichteten in einer Studie Männer mit $\mathrm{BPH}$, die über mögliche sexuelle Nebenwirkungen einer 5-ARI-Therapie aufgeklärt wurden, drei Mal häufiger über selbige als Männer, die nicht aufgeklärt wurden [Mondaini et al. J Sex Med 2007; 4: 1708-12]. Ob Patienten, die 5-ARI (als Mono- oder Kombinationstherapie) einnehmen, eine erhöhtes Risiko für ein hochgradiges Prostatakarzinom haben, bleibt derweil unklar. In der REDUCEStudie wurden in den Studienjahren 3 bis 4 zwar bei signifikant mehr Patienten unter Dutasterid Gleason-Score-Tumore 8-10 entdeckt als unter Placebo, dieser Unterschied bestätigte sich allerdings in keiner der Folgestudien.

Sebastian Lux

Symposium „Risk Stratification to Optimize the Management of Men With Symptomatic BPH at Risk of Progression" im Rahmen des 28. EAU-Kongresses in Mailand,

16. März 2013

Veranstalter: GlaxoSmithKline, München

\section{Prostatakarzinom: neuer Test zur Vorhersage des Rezidivrisikos}

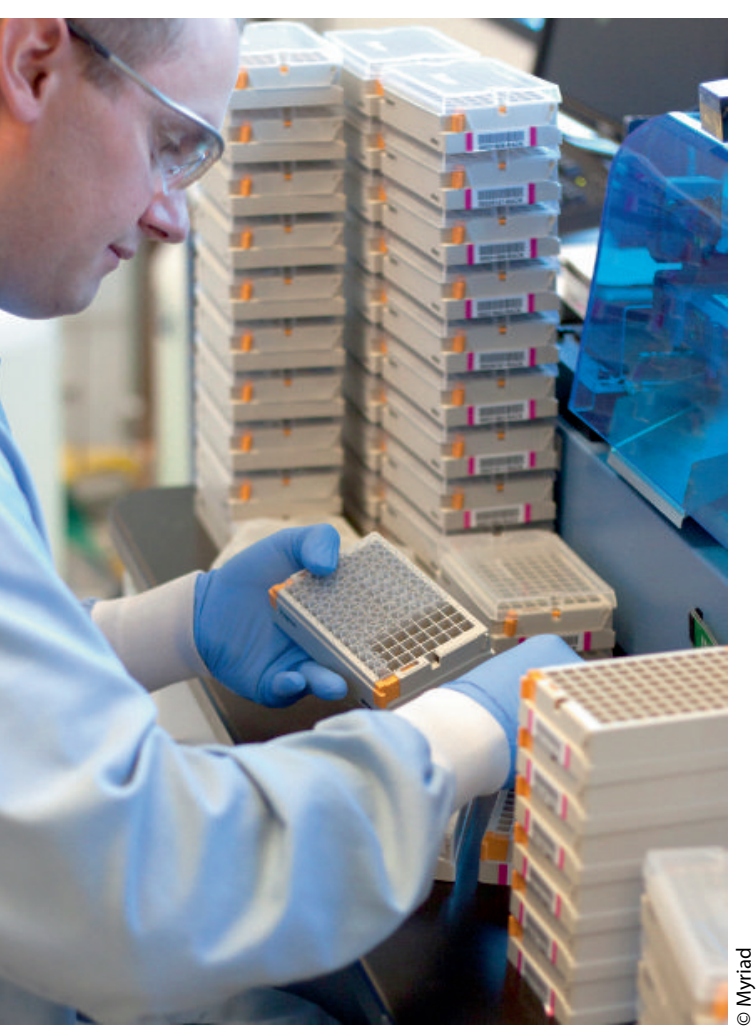

- Die Therapieentscheidung beim Prostatakarzinom wird vielfach aufgrund eines erhöhten PSA-Wertes und dem Gleason-Risikoscore getroffen. Ein genaueres prognostisches Werkzeug bietet der Test Prolaris ${ }^{\circledast}$, der anhand von 46 Genmustern (CCP-Score) die Aggressivität von Prostatakrebs vorhersagt und damit Grundlage für Behandlungs- und Überwachungsstrategien ist. Der Test erlaubt eine direkte molekulare Messung der Tumorbiologie. Indem der Aktivierungsgrad jener Gene bestimmt wird, die am Tumorwachstum beteiligt sind, kann der Krankheitsverlauf sehr genau prognostiziert werden. Der ermittelte CCP-Score kann dabei unterstützen vorherzusagen, welche Männer indolenten Prostatakrebs haben und damit eventuell für eine aktive Überwachung infrage kommen, und welche Männer aggressiven Prostatakrebs haben und von definitiver Behandlung wie Prostatektomie oder Strahlentherapie profitieren könnten.

Bisher wurden mehr als 1.500 Patienten in klinische Studien aufgenommen, weitere 2.000 Patienten nehmen derzeit an laufenden Untersuchungen mit Prolaris ${ }^{\circledR}$ teil beziehungsweise werden rekrutiert. Vier Studien bieten klinische Evidenz für den Einsatz des prognostischen Tests, der eine signifikant höhere prognostische Aussagekraft vorweist als alle derzeit verwendeten klinischen und pathologischen Variablen, erklärte Michael Brawer von Myriad Genetics, Salt Lake City/ USA.

Dr. Vincenzo Scattoni, Mailand, berichtete von frühen Erfahrungen seines Instituts. Er wies darauf hin, dass die Arbeit des Pathologen für den Gentest nicht zu unterschätzen sei, da beim Umgang mit der RNA sehr vorsichtig nach Anweisung vorgegangen werden müsse. Neueinsteigern riet er, erst wenige Proben einzusenden, das Ergebnis abzuwarten und dann weitere Fälle zu präparieren.

Dr. Ine Schmale

Pressekonferenz „EU launch of Prolaris ${ }^{\oplus}$ : A Gene Expression Test to Predict Cancer Aggressivness" im Rahmen des 28. EAU-Kongresses in Mailand,

17. März 2013

Veranstalter: Myriad, Martinsried 